\title{
SYMMETRY OF KNOTS AND CYCLIC SURGERY
}

\author{
SHICHENG WANG AND QING ZHOU
}

\begin{abstract}
If a nontorus knot $K$ admits a symmetry which is not a strong inversion, then there exists no nontrivial cyclic surgery on $K$. No surgery on a symmetric knot can produce a fake lens space or a 3-manifold $M$ with $\left|\pi_{1}(M)\right|=2$. This generalizes the result of Culler-Gordon-Luecke-ShalenBleiler-Scharlemann and supports the conjecture that no nontrivial surgery on a nontrivial knot yields a 3-manifold $M$ with $\left|\pi_{1}(M)\right|<5$.
\end{abstract}

\section{1}

Section 1 includes the statements of the main result and its corollaries; discussion of some examples and facts related to symmetry and cyclic surgery on knots; some terminology and theorems which are used in $\S 2$ (the proof of main result).

The known minimum positive integer $p$ such that a lens space $L(p, q)$ can be the result of a surgery on some nontrivial knot $K$ (the $(2,3)$ torus knot) is 5. It is reasonable to make the following

Conjecture. No nontrivial surgery on a nontrivial knot can yield a 3-manifold $M$ with $\left|\pi_{1}(M)\right|<5$.

This conjecture is related to the Property P Conjecture: No nontrivial surgery on a nontrivial knot can yield a 3-manifold $M$ with $\left|\pi_{1}(M)\right|=1$.

Gabai proved that the Property $\mathrm{P}$ Conjecture is true for satellite knots. $\mathrm{S}$. Bleiler and M. Scharlemann proved that the Property $\mathbf{P}$ Conjecture is true for nontrivial strongly invertible knots. M. Culler, C. M. Gordon, and J. Luecke and $\mathrm{P}$. Shalen proved that the Property $\mathrm{P}$ Conjecture is true for a nontrivial symmetric knot which is not strongly invertible. Hence it is reasonable first to prove the Conjecture above for satellite knots and symmetric knots.

In [W], using the result of [Ga and CGLS], it was proved that if a lens space (lens space can be replaced by 3-manifold with finite cyclic fundamental group) is obtained from a nontrivial surgery on a satellite knot, then the order of its fundamental group is not smaller than 23. So the conjecture is true for satellite knots.

The main result of this paper is the following

Theorem. If a nontorus knot $K$ admits a symmetry which is not a strong inversion, then there exists no nontrivial cyclic surgery on $K$.

Received by the editors June 18, 1989 and, in revised form, January 12, 1990.

1980 Mathematics Subject Classification (1985 Revision). Primary 57N10.

(C) 1992 American Mathematical Society $0002-9947 / 92 \$ 1.00+\$ .25$ per page 
This Theorem generalizes the result of M. Culler-C. M. Gordon-J. Luecke-P. Shalen above and supports the conjecture for symmetric knots.

Corollary 1. No surgery on a symmetric knot can produce a fake lens space.

Recently S. Bleiler and R. Litherland proved that if a nontrivial knot admits a strong inversion, then no surgery on $K$ yields real projective 3-space $R P^{3}$. Hence we have

Corollary 2. No nontrivial surgery on a nontrivial symmetric knot can produce a 3-manifold $M$ with $\pi_{1}(M)=\mathbf{Z}_{2}$.

Remark (1). The situation of torus knots was fully explored in the work of $\mathrm{L}$. Moser.

From the Theorem, one may think that a knot's having a symmetry works against its having a cyclic surgery. Such a conclusion is not quite right. Actually we have the

Proposition. If a satellite knot admits a cyclic surgery, then it must be strongly invertible and has no other symmetries.

Proof. In [W], Wang observed that if a satellite knot $K$ admits a cyclic surgery, then $K$ is braid in a regular neighborhood of a torus knot. Later $\mathrm{Wu}$, Bleiler, Litherland (see [Wu or BL]) proved that $K$ must actually be the $(2 p q \pm 1,2)$ cable of a $(p, q)$ torus knot. But it is not hard to see that an $(r, 2)$ cable of a torus knot is strongly invertible. On the other hand, our theorem says that there is no other symmetries.

As an example, Figure 1 below shows the symmetry of the Bailey-Rolfsen knot, the $(11,2)$ cable of the $(2,3)$ torus knot, which was the first example of a nontorus knot admitting a cyclic surgery (see [BR]).

By the Theorem above we thus have infinitely many examples of knots for which the only symmetry is a strong inversion.

The famous Fintushel-Stern knot [FS] admits a strong inversion (see Figure 2). Some surgeries on this knot yield the lens spaces $L(18)$ and $L(19)$ (see [FS]). By Corollary 2 of [W], the Fintushel-Stern knot is a hyperbolic knot. So we have an example of a hyperbolic knot for which the only symmetry is a strong inversion.

Remark (2). There is an obvious way to attach a band to the 2-bridge knot $b(3,1)$ or the 2-bridge link $b(4,1)$ to get the trivial knot (see Figure 3 ).

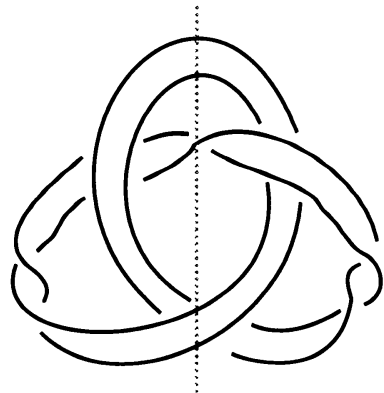

FIGURE 1 


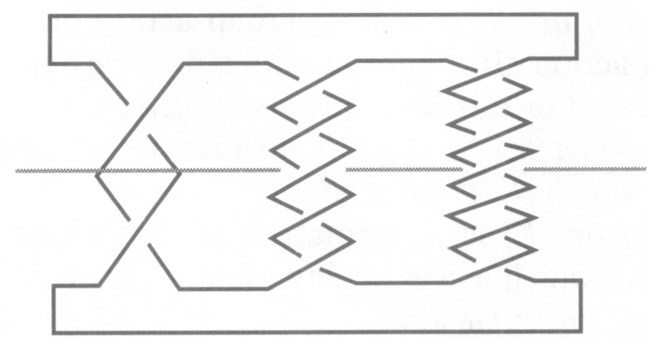

FIGURE 2

One may ask the question: Is there any other way (up to isotopy of $\left.(I \times I, \partial I \times I) \rightarrow\left(S^{3}, K\right)\right)$ to attach a band to $b(3,1)$ or $b(4,1)$ to get the trivial knot?

If the answer is no, then following the argument of Bleiler and Litherland, one can prove that there is no surgery on a strong invertible knot yields $L(3, q)$ or $L(4, q)$; therefore the conjecture for symmetric knots would be proved.

Note that there are different ways to attach a band to $b(5,1)$ to get the unknot. (See Figure 4.)

Let $K$ be a nontrivial knot in $S^{3}, N(K)$ denotes a tubular neighborhood of $K, N^{\circ}(K)$ the interior of $N(K)$, and $E(K)=S^{3}-N^{\circ}(K)$. Let $\lambda$ be the longitude, $\mu$ the meridian of $K$.

$S$ denotes a solid torus and $T$ a torus.

$L(p)$ is some lens space $L(p, q)$. A cable space is the complement of an open tubular neighborhood of a $(p, q)$ cable of the core of the solid torus $S$.
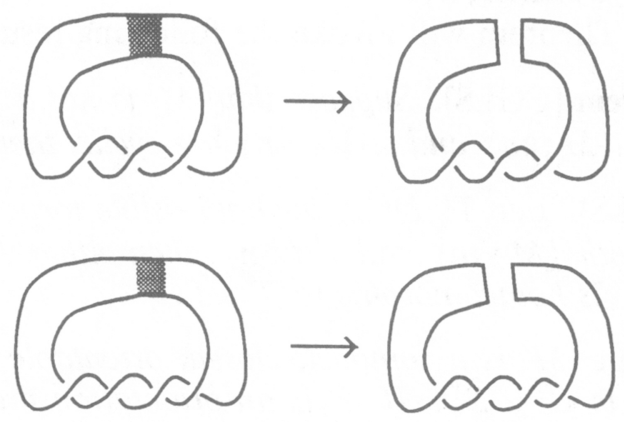

FIGURE 3
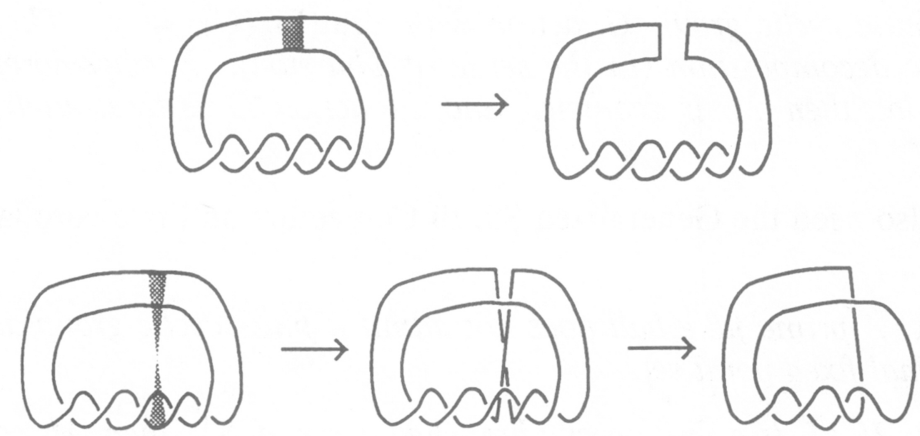

Figure 4 
A finite group action on $K$ is a finite group action on $S^{3}$ such that $K$ is invariant under this action. If a knot admits a finite group action, we say this knot is a symmetric knot or this knot has a symmetry.

A knot $K$ is amphicheiral if there is an orientation reversing homeomorphism of $S^{3}$ under which $K$ is invariant.

A strong inversion on $K$ is a $\pi$-rotation of $S^{3}$ whose axis meets $K$ in exactly two points and which leaves $K$ invariant. A knot $K$ admitting a strong inversion is a strongly invertible knot.

If a surgery on $K$ produces a 3-manifold $M$ with finite cyclic fundamental group, we call this surgery a cyclic surgery. If a closed 3-manifold has a finite cyclic fundamental group and is not a lens space, we call it a fake lens space. (The existence of fake lens spaces is unknown.)

A circle $C$ in $L(p)$ is standard, if $L(p)-N^{\circ}(C)$ is a solid torus.

Let $G$ be a finite group which acts on $M$. For any $g \in G, \operatorname{Fix}(g)$ is the fixed point set of $g$ and $\operatorname{Fix}(G)=\bigcup_{g \in G \text {-\{id\} }} \operatorname{Fix}(g)$. We say that $G$ acts freely on $M$, or the action $G$ is free, if $\operatorname{Fix}(G)=\varnothing$.

Let $M$ be a compact, connected, irreducible, oriented 3-manifold with a torus boundary component $T$. A nontrivial isotopy class of simple closed curves on $T$ is called a slope. In the case that $M$ is a knot complement, we shall always assume that the slope $m$ is not the meridian $\mu$. For any slope $m$ on $T,(M ; m)$ is constructed by attaching a solid torus $S$ to $M$ so that the slope $m$ bound a disk in $S$. For a knot complement $E(K),(E(K) ; m)$ is simply denoted by $(K ; m)$.

If $m$ and $n$ are two slopes on $T$, we denote their (minimal) geometric intersection number by $\Delta(m ; n)$.

The proof of the Theorem will invoke the following results.

Cyclic surgery theorem [CGLS]. Suppose that $M$ is not a Seifert fibered space and $\partial M=T$. If $\pi_{1}(M ; m)$ and $\pi_{1}(M ; n)$ are cyclic, then $\Delta(m ; n) \leq 1$.

Theorem 2.4.4 [CGLS]. Let $T^{\prime}$ be an incompressible torus in $\partial M-T$. If $T^{\prime}$ is compressible in both $(M ; m)$ and $(M ; n)$, then either $\Delta(m ; n) \leq 1 ; M$ is a cable space; or $M$ is homeomorphic to $T \times I$.

[MY, MSY]. Suppose $M$ is a compact, closed, orientable 3-manifold and no prime factor of $M$ is $S^{2} \times S^{1}$. If $G$ is an orientation preserving finite cyclic group action on $M$, then there is a G-equivariant prime factorization of $M$.

[T] (see also [SCK and Z]). Let $M$ be an irreducible, closed 3-manifold which admits a finite cyclic group $G$ action with $\operatorname{dim}(\operatorname{Fix}(G))=1$. Then $M$ has a geometric decomposition (in the sense of Thurston). Furthermore, if $M$ is also atoroidal, then $M$ is geometric and the action $G$ is by isometries of this geometry.

[MB] We also need the Generalized Smith Conjecture and two corollaries of its proof:

Corollary 1. A prime fake ball does not admit a finite cyclic group action with 1-dimensional fixed point set.

Corollary 2. If $M$ is a finite cyclic branched cover of $S^{3}$, then $M$ contains no fake ball. 
An involution has 1-dimensional fixed point set if and only if it is an orientation preserving involution with fixed points.

Let us list the useful properties of orientation preserving involutions with fixed points on lens spaces $L(p, q)$ (see [HR]).

Let $L(p, q)=U \cup V$, where $U, V$ are two solid tori. For our purpose, we describe involutions with 1-dimensional fixed point set on $L(p, q)$ as the following three types.

Type (A). Both $U$ and $V$ are invariant under the involution, however the orientation of the core (center line) of each solid torus is reversed under the involution. The orbit space is $S^{3}$.

Type (B). Both $U$ and $V$ are invariant under the involution, furthermore the orientation of the core of each solid torus is preserved under the involution. Any component of the fixed point set is the core of $U$ or $V$. In particular, any component of the fixed point set is a standard circle in $L(p, q)$.

Type (C). $U$ and $V$ are interchanged under the involution. The lens space $L(p, q)$ admits this type involution only if $q^{2} \equiv 1 \bmod p$. The orbit space is $L(p /(p, q+1),(q+1) /(p, q+1))$ or $L(p /(p, q-1),(q-1) /(p, q-1))$.

Two involutions on $L(p, q)$ are strongly equivalent if they are conjugate by a diffeomorphism which is isotopic to the identity.

Remark. In [HR], there are five types of involution $(\mathrm{A}),(\mathrm{B}),\left(\mathrm{B}^{\prime}\right),(\mathrm{C}),\left(\mathrm{C}^{\prime}\right)$. Our type (B) (resp. (C)) is the union of the types (B) and $\left(\mathrm{B}^{\prime}\right)$ (resp. (C) and $\left.\left(C^{\prime}\right)\right)$ in [HR]. In our definition, two involutions of the same type are not necessarily strongly equivalent.

[HR]. Every orientation preserving involution with fixed points on $L(p, q)$ is strongly equivalent to an involution belonging to one of the three types above. An involution of type (C) is not of type (A) or (B) if and only if $q \not \equiv \pm 1 \bmod p$.

In this section we give the proof of the Theorem and Corollary 1.

The proof of the Theorem is contained in Propositions 1, 2 and 3 below.

Proposition 1. If $K$ is not a torus knot and $K$ admits a cyclic group action $G=\left\{\eta \mid \eta^{n}=\mathrm{id}\right\}$ such that $\operatorname{Fix}(G) \neq \varnothing$, and $n>2$, then $\pi_{1}((K ; m)) \neq \mathbf{Z}_{p}$ for any $p$.

Proof. Suppose that $(K ; m)=E(K) \cup S$ has a finite cyclic fundamental group.

If $\eta$ is orientation reversing, then $K$ is amphicheiral. By [CGLS], $\pi_{1}(K, m)$ $\neq \mathbf{Z}_{p}$.

So we may assume that $\eta$ is orientation preserving. Then $\left.\eta\right|_{\partial E(K)}$ is orientation preserving and its order is larger than 2 .

By [H], for any periodic map $h$ on torus $T$, there is a basis for $H_{1}(T)=$ $\pi_{1}(T)$ with respect to which the matrix representation of $h_{*}$ is one of the following seven:

$$
\left(\begin{array}{cc}
1 & 0 \\
0 & -1
\end{array}\right)\left(\begin{array}{cc}
1 & 0 \\
1 & -1
\end{array}\right)\left(\begin{array}{cc}
-1 & 0 \\
0 & -1
\end{array}\right)\left(\begin{array}{cc}
0 & 1 \\
-1 & 1
\end{array}\right)\left(\begin{array}{cc}
0 & 1 \\
-1 & -1
\end{array}\right)\left(\begin{array}{cc}
0 & -1 \\
1 & 0
\end{array}\right)\left(\begin{array}{ll}
1 & 0 \\
0 & 1
\end{array}\right)
$$


A map corresponding to one of the first two matrices is not orientation preserving because the determinants are -1 . The map corresponding to the third one has fixed points, so does $\left(\left.\eta\right|_{\partial E(K)}\right)^{2}$, and $\left(\left.\eta\right|_{\partial E(K)}\right)_{*}^{2}=$ id. This will imply that $\eta$ has order 2 . The next three matrices have no eigenvectors. Since the longitude class must be invariant under $\eta,\left.\eta\right|_{\partial E(K)}$ can not correspond any one of those three matrices. So $\left.\eta\right|_{\partial E(K)}$ must be the last matrix. By the well-known fact, $\left.\eta\right|_{\partial E(K)}$ is isotopic to the identity and $G$ acts freely on $\partial E(K)$.

Since $G$ acts freely on $\partial E(K), \operatorname{Fix}(G)$ either lies in $E^{\circ}(K)$ or coincides with the core of $N(K)$. Because $K$ is a nontrivial knot, the Smith conjecture rules out the possibility that $\operatorname{Fix}(G)$ is the core of $N(K)$. Hence $\operatorname{Fix}(G)$, denote it by $C$, is a standard circle in $S^{3}$, and lies in $E(K)$.

Since $\left.\eta\right|_{\partial E(K)}$ is isotopic to the identity, the action $G=\left\{\eta \mid \eta^{n}=\mathrm{id}\right\}$ on $E(K)$ can also be extended to an action on $(K ; m)=E(K) \cup S$, which is denoted by $G_{1}=\left\{\eta_{1} \mid \eta_{1}^{n}=\mathrm{id}\right\}$. The restriction of $G_{1}$ on $E(K)$ coincides with the restriction of $G$ on $E(K)$. If the action $G_{1}$ is not fixed point free on $S$, then the fixed point set of $G_{1}$ must be the core of $S$. Hence there is $\eta_{1}^{i} \in G_{1}$ such that its fixed point is the core of $S$.

Let us temporarily assume that $E(K) \cup S$ is a lens space $L(p)$.

Let $q: S^{3} \rightarrow L(p)$ be the universal cover.

Let $\widetilde{S}=q^{-1}(S)$. Since $\pi_{1}(S) \rightarrow \pi_{1}(L(p))$ is onto, $\widetilde{S}$ has only one component. By covering space theory, there is a $\tilde{\eta}$, a lift of $\eta_{1}^{i}$, with $\operatorname{Fix}(\tilde{\eta}) \neq \varnothing$. The fact that $q(\operatorname{Fix}(\tilde{\eta})) \subset \operatorname{Fix}\left(\eta^{i}\right)$ implies that $\operatorname{Fix}(\tilde{\eta}) \subset$ the core of $\tilde{S}$. Since $\tilde{\eta}$ is of finite order and orientation preserving, by the Smith conjecture we have Fix $(\tilde{\eta})$ equal to the core of $\widetilde{S}$, and the core of $\widetilde{S}$ is unknotted in $S^{3}$. Hence $E(K)=q\left(S^{3}-N^{\circ}(\widetilde{S})\right)$ is a solid torus. It is a contradiction. So $G_{1}$ acts freely on $S$ and we have

$$
\operatorname{Fix}\left(G_{1}\right)=\operatorname{Fix}(G)=C .
$$

Lemma 1. Let $G=\left\{g \mid g^{n}=\mathrm{id}\right\}$ act on $L(p)$. If $n>2$ and $\operatorname{Fix}(G) \neq \varnothing$, then any component $C$ of $\operatorname{Fix}(G)$ is standard.

Proof. We first impose the two conditions $\operatorname{Fix}(g) \neq \varnothing$ and $n \neq 4$ on $G$.

By [T], there is a spherical geometry on $L(p)$ such that the action $G$ is as a group of isometries. So every component $C \subset \operatorname{Fix}(G)$ is a geodesic.

Let $q: S^{3} \rightarrow L(p)$ be the universal cover and $\Xi=\left\{h \mid h^{p}=\right.$ id $\}$ be the deck transformation group.

Pick a component $\tilde{C} \subset q^{-1}(C)$, then $\tilde{C} \subset \operatorname{Fix}(\tilde{g})$ for some lift $\tilde{g}$ of $g$. Now $\widetilde{C}$ is a great circle on $S^{3} \subset R^{4}$ and $\tilde{g}, h$ are orientation preserving orthogonal maps on $R^{4}$.

The fact that the great circle $\widetilde{C} \subset \operatorname{Fix}(\tilde{g}) \subset \operatorname{Fix}\left(\tilde{g}^{2}\right)$ implies that under a suitable basis, $\tilde{g}^{2}$ corresponds to a matrix

$$
\left(\begin{array}{cccc}
1 & 0 & 0 & 0 \\
0 & 1 & 0 & 0 \\
0 & 0 & \cos \alpha & -\sin \alpha \\
0 & 0 & \sin \alpha & \cos \alpha
\end{array}\right)
$$

where $0 \leq \alpha<2 \pi$ is some rotation angle on an invariant plane of $\tilde{g}^{2}$. The fact that the order of $g^{2}$ is not 2 (since the order of $g$ is not 4) implies that the order of $\tilde{g}^{2}$ is not 2; therefore the order of this matrix is not 2. So $\alpha$ is 
not 0 or $\pi$. From linear algebra, it is not hard to see that $\tilde{g}^{2}$ has exactly two invariant planes $V_{1}, V_{2}$. We may assume that $\widetilde{C}=V_{1} \cap S^{3}$. We will show that $V_{1}$ is invariant under $\Xi$, hence $\widetilde{C}$ and the solid torus $S^{3}-N^{\circ}(\widetilde{C})$ are invariant under $\Xi$. So $S^{3}-N^{\circ}(C)$, as the quotient of $S^{3}-N^{\circ}(\widetilde{C})$, is a solid torus and $C$ is a standard circle in $L(p, q)$.

By covering space theory, we have $\tilde{g} h=h^{i} \tilde{g}$ for some $i$, i.e. we have $h^{i}=\tilde{g} h \tilde{g}^{-1}$. This means that $h^{i}$ is a generator of $\Xi$.

Now under a suitable basis, $h$ has a matrix

$$
H=\left(\begin{array}{cccc}
\cos \beta & -\sin \beta & 0 & 0 \\
\sin \beta & \cos \beta & 0 & 0 \\
0 & 0 & \cos \gamma & -\sin \gamma \\
0 & 0 & \sin \gamma & \cos \gamma
\end{array}\right)
$$

where $0 \leq \beta, \gamma<2 \pi$ are some angle of rotations. Since $h$ acts freely on $S^{3} \subset R^{4}$, we have

$$
H \neq\left(\begin{array}{cccc}
1 & 0 & 0 & 0 \\
0 & 1 & 0 & 0 \\
0 & 0 & -1 & 0 \\
0 & 0 & 0 & -1
\end{array}\right)
$$

under any basis of $R^{4}$.

Case (1). $\beta \neq \gamma$, or equivalently, the characteristic polynomial of $h$ is not a perfect square. (Now the corresponding lens space is $L(p ; q), q \neq 1$.) Now again from linear algebra we know that $h$ has exactly two invariant planes $U_{1}$ and $U_{2}$ and both of them are invariant under $h^{i}$ for any $i$. The fact that $\tilde{g} h=h^{i} \tilde{g}$ impiies that $\tilde{g}\left(U_{1}\right)$ is $U_{1}$ or $U_{2}$. Anyway we get $\tilde{g}^{2}\left(U_{i}\right)=U_{i}$ for $i=1,2$. So $V_{1}$ is $U_{i}$ for some $i=1$ or 2 . In particular $V_{1}$ is invariant under $\Xi$. Therefore $\widetilde{C}$ is invariant under $\Xi$.

Case (2). $\beta=\gamma$, or equivalently, the characteristic polynomial of $h$ is a perfect square (the corresponding lens space is $L(p ; 1))$. Now $h$ has infinitely many invariant planes. The important fact is that the action of $h$ on every invariant plane is a rotation by the same angle $\beta$. From this fact and the fact that if $U$ is an invariant plane of $h$, so is $\tilde{g}(U)$, it is not hard to see one of the following is true:

$$
\tilde{g} h=h \tilde{g}, \quad \tilde{g} h=h^{-1} \tilde{g} .
$$

In either case, we have $\tilde{g}^{2} h=h \tilde{g}^{2}$.

Now $\widetilde{C} \subset \operatorname{Fix}\left(\tilde{g}^{2}\right)$ implies $h(\widetilde{C}) \subset \operatorname{Fix}\left(h \tilde{g}^{2} h^{-1}\right)=\operatorname{Fix}\left(\tilde{g}^{2}\right)$.

Both $\widetilde{C}$ and $h(\widetilde{C})$ belong to $\operatorname{Fix}\left(\tilde{g}^{2}\right)$. Since $\tilde{g}^{2} \neq \mathrm{id}$, by well-known properties of orthogonal maps, we must have $h(\widetilde{C})=\widetilde{C}$. Therefore $\widetilde{C}$ is invariant under $\Xi$.

Now we first remove the condition $n \neq 4$. If $n=4$, pick any component $C \subset \operatorname{Fix}(g) \subset \operatorname{Fix}\left(g^{2}\right) . g^{2}$ is an orientation preserving involution with fixed points. By [HR] every orientation preserving involution with fixed points belongs to one of the three types (A), (B) or (C) defined above. For any involution of type (B), each component of the fixed point set is a standard circle. If $C$ is not standard, then $g^{2}$ belongs to the one of the type $(A)$ or $(C)$. However, in the mapping class group of $L(p)$, neither of them is a power of any mapping 
class; therefore neither of them contains $g^{2}$. This is a contradiction. So any component $C$ of $\operatorname{Fix}(g)$ is a standard circle.

Finally, let us remove the condition $\operatorname{Fix}(g) \neq \varnothing$. If there is any $C \subset \operatorname{Fix}(G)$, then $C \subset \operatorname{Fix}\left(g^{i}\right)$ for some $i$. If $C$ is not standard, by the fact we have proved, $g^{i}$ is of order 2 . By the argument in the last paragraph, the only possibility is $i=1$. So $g$ is of order 2 .

Lemma 1 is proved.

Now we show that $E(K) \cup S-N^{\circ}(C)$ is a homotopy solid torus without the assumption that $E(K) \cup S$ is a lens space.

Let $L_{1}=E(K) \cup S$. By [MY, MSY], there is a $G$ equivariant prime decomposition of $L_{1}$. Then we can replace all fake balls produced from the decompositon by standard balls to get a 3-manifold $L_{2}$, and the action $\left.G_{1}\right|_{L_{1} \text {-fake balls }}$ can be extended to an action $G_{2}$ on $L_{2}$. Since $\pi_{1}\left(L_{2}\right)=\pi_{1}\left(L_{1}\right)=\mathbf{Z}_{p}$ and $L_{2}$ contains no fake ball, $L_{2}$ is irreducible. Since no fake ball hits $C$ by [MB], $\operatorname{Fix}\left(G_{2}\right)=\operatorname{Fix}\left(G_{1}\right)=C$. Since the oriented irreducible closed 3-manifold $L_{2}$ admits the group $G_{2}$ action with 1-dimensional fixed point set, $L_{2}$ has a geometric decomposition by [T]. Then $L_{2}$ must support the geometry of $S^{3}$ (see [S]); therefore $L_{2}$ is a lens space. By lemma $1, L_{2}-N^{\circ}(C)$ is a solid torus. Since no fake ball hits $C$, we may assume that no fake ball hits $N(C)$. Then $L_{1}-N^{\circ}(C)$ is a homotopy solid torus.

Let $R=E(K)-N^{\circ}(C)$. The restriction of the cyclic group $G$ acts freely on $R$, and $\partial N(C)$ and $\partial E(K)$ are invariant under $G$.

Let $M=R / G$. Then $M$ has two boundary components $T^{\prime}=\partial N(C) / G$ and $T=\partial E(K) / G$. In the covering $R \rightarrow M$, the connectness of $\partial E(K)$ implies that the linking number of $C$ and $K$ is not zero. So $R$ is irreducible; therefore $M$ is irreducible.

The images of the slopes $m$ and $\mu$ on $\partial E(K)$ are two slopes $m^{\prime}$ and $n^{\prime}$ on $T$. (Since both $m$ and $\mu$ bound meridian discs.) Then

$$
\left(M ; m^{\prime}\right)=(R ; m) / G_{1}=\left[L(p)-N^{\circ}(C)\right] / G_{1}
$$

is a homotopy solid torus.

Also we have

$$
\left(M ; n^{\prime}\right)=(R ; \mu) / G=\left[S^{3}-N^{\circ}(C)\right] / G
$$

is a solid torus.

Since $C$ is unknotted and $K$ is not a torus knot, $R$, and hence $M$ is not a cable space or $T \times I$. Now $T^{\prime}$ is compressible in both $\left(M ; m^{\prime}\right)$ and $\left(M ; n^{\prime}\right)$, hence by [CGLS, 2.4.4], $\Delta\left(m^{\prime} ; n^{\prime}\right) \leq 1$.

There are $n$ parallel circles in $\partial E(K)$ as the preimage of $m^{\prime}$. There are $n$ parallel circles in $\partial E(K)$ as the preimage of $n^{\prime}$. Every intersection point between $m^{\prime}$ and $n^{\prime}$ creates $n^{2}$ intersection points between these two sets of parallel circles. Remember that the degree of the covering map is $n$. So we have

$$
\Delta\left(m^{\prime} ; n^{\prime}\right)=\frac{n^{2}}{n} \Delta(m ; \mu)=n \Delta(m ; \mu)>1,
$$

which is a contradiction. This shows that $(K ; m)$ can not have cyclic fundamental group.

Propositon 1 is proved. 
Remark. From the last part of proof of Proposition 1, we see that: Suppose a finite cyclic group $G$ acts on a nontorus knot $K$, with $\operatorname{Fix}(G) \neq \varnothing, \operatorname{Fix}(G) \cap$ $K=\varnothing$. If $(K, m)$ has a finite cyclic fundamental group, the $(K, m)-\operatorname{Fix}(G)$ is not a homotopy solid torus. This will be used in the proof of the Proposition 3.

Proposition 2. If $K$ is not a torus knot and $K$ admits a free action of some nontrivial finite group $G$, then $\pi_{1}(K ; m) \neq \mathbf{Z}_{p}$ for any integer $p$.

Proof. We may suppose that $G$ is a cyclic group $\mathbf{Z}_{n}$ which is generated by $\eta$. Since the restriction of $\eta$ on the boundary of $E(K)$ is fixed point free and orientation preserving, as discussed in the proof of Proposition 1, we see that $\left.\eta\right|_{\partial E(K)}$ is isotopic to the identity. So the restriction of the action $G=$ $\left\{\eta \mid \eta^{n}=\right.$ id $\}$ on $E(K)$ can be extended to an action $G_{1}=\left\{\eta_{1} \mid \eta_{1}^{n}=\right.$ id $\}$ on $(K ; m)=E(K) \cup S$.

Suppose $(K ; m)=E(K) \cup S$ has finite cyclic fundamental group. Let $q: N^{3} \rightarrow E(K) \cup S$ be the universal covering, and $\Xi=\left\{\tau \mid \tau^{p}=\right.$ id $\}$ be the deck transformation group of this covering, here $N^{3}$ is a homotopy 3-sphere. By the same argument in the proof of Proposition 1, we know that $q^{-1}(S)$ is a solid torus $\tilde{S}$ which is invariant under the deck transformation group. Obviously the core of $\widetilde{S}$ is the preimage of the core of $S$ under $q$.

By the same reason used in the proof of Proposition 1, the $G_{1}$ action is still free.

$N^{3}$ is the universal covering of $(K ; m) / G_{1}$. The preimage of the solid torus $S / G_{1}$ is $\tilde{S}$ which is connected. Hence $\pi_{1}\left(S / G_{1}\right) \rightarrow \pi_{1}\left((K ; m) / G_{1}\right)$ is onto. Therefore $\pi_{1}\left((K ; m) / G_{1}\right)$ is cyclic.

The coverings $E(K) \cup S \rightarrow[E(K) \cup S] / G_{1}$ and $S^{3} \rightarrow S^{3} / G$ coincide on $E(K) \rightarrow E(K) / G=M$, say. In $E(K) \cup S$, the slope $m$, as the boundary of meridian disk of $S$, is homeomorphic to a slope $m^{\prime}$ on $\partial M$ under the covering map and

Similarly,

$$
(K ; m) / G_{1}=\left(M ; m^{\prime}\right) .
$$

$$
(K ; \mu) / G=S^{3} / G=\left(M ; n^{\prime}\right) .
$$

Here the slope $n^{\prime}$ on $\partial M$ is the image of the slope $\mu$ under the covering map.

Since $K$ is not a torus knot, $M$ is not a Seifert fibered space. Both $\left(M ; n^{\prime}\right)$ and $\left(M ; m^{\prime}\right)$ have cyclic fundamental group. By the cyclic surgery theorem, we should have $\Delta\left(m^{\prime} ; n^{\prime}\right) \leq 1$. As discussed in the proof of Proposition 1, we have

$$
\Delta\left(m^{\prime} ; n^{\prime}\right)=\frac{n^{2}}{n} \Delta(m ; \mu)=n \Delta(m ; \mu)>1,
$$

which is a contradiction.

Proposition 2 is proved.

Proposition 3. If $K$ is not a torus knot and $K$ admits an involution $\eta$ with 1-dimensional fixed point set, which is not a strong inversion, then there is no cyclic surgery on $K$.

Proof. When $p=1$, Proposition 3 is true by [CGLS]. We may assume that $p>1$.

Suppose $\pi_{1}(E(K) \cup S)=\mathbf{Z}_{p}$ for some $p$. The fact that the action $\eta$ is not a strong inversion implies that $\operatorname{Fix}(\eta) \cap \partial E(K)=\varnothing$ and $\left.\eta\right|_{\partial E(K)}$ is isotopic to the 
identity. As in the proof of Proposition 1, the action $\left.G\right|_{E(K)}$ can be extended to an involution $\eta_{1}$ on $E(K) \cup S$ and the fixed point set of $\eta_{1}$ lies in $E^{\circ}(K)$.

We have $S^{3}=S^{3} / \eta=[E(K) / \eta] \cup[N(K) / \eta]$. Since $\eta$ acts freely on $N(K)$, $N(K) / \eta$ is a solid torus. So $E(K) / \eta=E(K) / \eta_{1}$ is a knot complement $E(\bar{K})$ for some knot $\bar{K}$. Let $\bar{S}=S / \eta_{1}$. We have a double branched cover $p: E(K) \cup$ $S \rightarrow\left[E(K) / \eta_{1}\right] \cup\left[S / \eta_{1}\right]=E(\bar{K}) \cup \bar{S}$.

Let $q: S^{3} \rightarrow S^{3} / \eta$ be the quotient map. Let $\bar{F}$ be a Seifert surface for $\bar{K}$, transverse to $q(\operatorname{Fix}(\eta))$. Let $F=q^{-1}(\bar{F})$. Then $\partial F=q^{-1}(\bar{K})=K$, and $\eta(F)=F$. Then $\eta_{1}(\lambda)=\lambda$ and $\bar{\lambda}=p(\lambda)$ is a longitude of $\bar{K}$. Now the map $\left.p\right|_{\lambda}$ from $\lambda$ to $\bar{\lambda}$ is two to one. Let $\bar{D}$ be a meridian disk of $\bar{S}$. Since $\eta_{1}$ acts freely on $S, p^{-1}(\bar{D})$ is two disjoint meridian disks $D_{1}$ and $D_{2}$ of $S$. We have

$$
p^{-1}(\bar{\lambda} \cap \partial \bar{D})=p^{-1}(\bar{\lambda}) \cap p^{-1}(\partial \bar{D})=\lambda \cap\left(\partial D_{1} \cup \partial D_{2}\right)=\left(\lambda \cap \partial D_{1}\right) \cup\left(\lambda \cap \partial D_{2}\right) .
$$

Since $\eta_{1}$ is an orientation preserving free involution on $\partial E(K)$ and $\eta_{1}\left(\partial D_{1}\right)$ $=\partial D_{2}$. We have

$$
\Delta\left(p^{-1}(\bar{\lambda}) ; p^{-1}(\partial \bar{D})\right)=2 \Delta(\bar{\lambda} ; \partial \bar{D})
$$

and

$$
\Delta\left(\lambda ; \partial D_{1}\right)+\Delta\left(\lambda ; \partial D_{2}\right)=2 \Delta\left(\lambda ; \partial D_{1}\right) .
$$

So finally we get

$$
\Delta(\bar{\lambda} ; \partial \bar{D})=\Delta\left(\lambda ; \partial D_{1}\right)
$$

Let $L=E(K) \cup S, \bar{L}=E(\bar{K}) \cup \bar{S}$, then from the assumption that $\pi_{1}(L)=\mathbf{Z}_{p}$ and $(*)$, we have $H_{1}(L, \mathbf{Z})=H_{1}(\bar{L}, \mathbf{Z})=\mathbf{Z}_{p}$. By [MY, MSY], there is a $G$ equivariant prime decomposition of $L$. Replacing all fake balls produced from the decomposition by standard balls to get a 3-manifold $L^{\prime}$, and the involution $\left.\eta_{1}\right|_{L \text {-fake balls }}$ can be extended to an involution $\eta^{\prime}$ on $L^{\prime}$. The facts that $L^{\prime}$ contains no fake ball and $\pi_{1}\left(L^{\prime}\right)=\pi_{1}\left(L_{1}\right)=\mathbf{Z}_{p}$ imply that $L^{\prime}$ is irreducible. Since no fake ball hits $\operatorname{Fix}\left(\eta_{1}\right)$ by $[\mathrm{MB}]$, Fix $\left(\eta_{1}\right)=\operatorname{Fix}\left(\eta^{\prime}\right)$. The oriented irreducible closed 3-manifold $L^{\prime}$ admits the involution $\eta^{\prime}$ with 1-dimensional fixed point set implies that $L^{\prime}$ has a geometric decomposition by [T]. Then $L^{\prime}$ must support geometry of $S^{3}$ (see [S]); therefore $L^{\prime}=L(p, q)$ for some $q$. Now let $\bar{L}^{\prime}=L^{\prime} / \eta^{\prime}$, then $\bar{L}^{\prime}$ and $\bar{L}$ are homotopy equivalent (since the former is obtained from the latter by trading homotopy 3-balls). So we get a double branched cover $L(p, q) \rightarrow \bar{L}^{\prime}$ with $H_{1}\left(\bar{L}^{\prime}\right)=\mathbf{Z}_{p}$.

What type of involution does $\eta^{\prime}$ belong to ?

Suppose that $\eta^{\prime}$ is an involution of type (C) and is not an involution of type (B) or (A). Then we have (1) $q^{2} \equiv 1 \bmod p$, (2) $q \not \equiv \pm 1 \bmod p$, and (3) the orbit space $\bar{L}^{\prime}$ is

$$
L\left(\frac{p}{(p, q+1)}, \frac{q+1}{(p, q+1)}\right) \text { or } L\left(\frac{p}{(p, q-1)}, \frac{q-1}{(p, q-1)}\right) .
$$

From the first homology of the $\bar{L}^{\prime}$ we know that

$$
p=\frac{p}{(p, q+1)} \text { or } \frac{p}{(p, q-1)} \text {. }
$$

This implies that $(p, q-1)$ or $(p, q+1)$ is 1 . Suppose $(p, q-1)=1$. By condition (1), we have $(q+1)(q-1)=q^{2}-1=m p$, for some integer $m$, and 
therefore $(p, q-1)=1$ implies that $p$ is a divisor of $q+1$, i.e. $q \equiv-1 \bmod p$. Similarly, if $(p, q+1)=1$, we have $q \equiv 1 \bmod p$. Hence condition (2) is not satisfied contradicting our assumption.

Suppose that $\eta^{\prime}$ is an involution of type (B); then the complement of the fixed point set of $\eta^{\prime}$ in $L^{\prime}$ is a solid torus. From the relation between $L_{1}$ and $L^{\prime}$, it follows that the complement of the fixed point set of $\eta_{1}$ in $L_{1}$ is a homotopy solid torus. The argument in the proof of Proposition 1 rules out this possibility. (See the remark after the proof of Proposition 1.)

Suppose that $\eta^{\prime}$ is an involution of type (A); then we have a double branched cover $L(p, q) \rightarrow S^{3}=\bar{L}^{\prime}$. This implies that $p=1$, contradicting our assumption that $p>1$.

This completes the proof of Proposition 3; therefore we have finished the proof of the Theorem.

Proof of Corollary 1. We need only to show that if $\eta$ is a strong inversion of $K$, then $(K ; m)$ is not a fake lens space for any $m$.

Under any basis of $\partial E(K)=T,\left(\left.\eta\right|_{T}\right)_{*}$ has matrix

$$
\left(\begin{array}{cc}
-1 & 0 \\
0 & -1
\end{array}\right)
$$

and the involution $\left.\eta\right|_{E(K)}$ can be extended to an involution $\eta_{1}$ on $E(K) \cup S$. Since $\eta$ is a $\pi$-rotation on $S^{3}$, we have $S^{3}=S^{3} / \eta=[E(K) / \eta] \cup[N(K) / \eta]$. Since $N(K) / \eta$ is a 3-ball $B^{3}, E(K) / \eta=E(K) / \eta_{1}$ is a 3-ball $B^{3}$. Since $S / \eta_{1}$ is also a 3-ball $B^{3}$, we have a double branched cover

$$
p: E(K) \cup S \rightarrow\left[E(K) / \eta_{1}\right] \cup\left[S / \eta_{1}\right]=B^{3} \cup B^{3}=S^{3} .
$$

By [MB], as the double branch cover of $S^{3}, E(K) \cup S$ contains no fake ball. If $E(K) \cup S$ has a finite cyclic fundamental group, then $E(K) \cup S$ is irreducible. Again, using the result of Thurston, $E(K) \cup S$ is a lens space.

We would like to express our gratitute to Professor R. D. Edwards for his help, and the referee for his valuable comments.

\section{REFERENCES}

[BR] J. Bailey and D. Rolfsen, An unexpected surgery construction of lens space, Pacific J. Math. 71 (1977), 295-298.

[BL] S. Bleiler and R. Litherland, Lens spaces and Dehn surgery, Proc. Amer. Math. Soc. 107 (1989), 1127-1131.

[BS] S. Bleiler and M. Scharlemann, A projective plane with three critical points is standard MSRI, preprint.

[CGLS] M. Culler, C. M. Gordon, J. Luecke and P. B. Shalen, Dehn surgery on knots, Ann. of Math. (2) 125 (1987), 237-300.

[FS] R. Fintushel and R. Stern, Constructing lens spaces by surgery on knots, Math. Z. 175 (1980), 33-51.

[Ga] D. Gabai, Foliation and surgery on knots, Bull. Amer. Math. Soc. 15 (1986), 83-86.

[H] J. Hempel, 3-manifolds, Princeton Univ. Press, Princeton, N. J., 1976.

[HR] C. Hodgson and J. H. Rubinstein, Involutions and isotopies of lens spaces, Lecture Notes in Math., vol. 1144, Springer-Verlag, 1983.

[M] L. Moser, Elementary surgery along torus knots, Pacific J. Math. 38 (1971), 734-745.

[MB] J. Morgan and H. Bass, The Smith conjecture, Academic Press, New York, 1984. 
[MY] W. Meeks and S. Yau, Topology of three-dimensional manifolds and the embedding problems in minimal surface theory, Ann. of Math. (2) 112 (1980), 441-484.

[MSY] W. Meeks, L. Simon and S. Yau, Embedded minimal surfaces, exotic sphere, and manifolds with positive Ricci curvature, Ann. of Math. (2) 116 (1982), 621-659.

[R] D. Rolfsen, Knots and links, Publish or Perish, Cambridge, Mass., 1976.

[S] P. Scott, The geometry of 3-manifolds, Bull. London Math. Soc. 15 (1983), 401-487.

[SCK] T. Soma, K. Chshika and S. Kojima, Towards a proof of Thurston's geometrization theory for orbifolds, Hyperbolic Geometry and Three Manifolds, Res. Inst. Math. Sci., Kokyuroku 568 (1986), 1-72.

[T] W. Thurston, Three-manifold with symmetry, preprint.

[W] S. Wang, Cyclic surgery on knots, Proc. Amer. Math. Soc. 107 (1989), 1091-1094.

[Wu] Y. Wu, Topology Appl. 36 (1990), 205-208.

[Z] Q. Zhou, 3-dimensional geometric cone structures, Thesis, UCLA, 1990.

Department of Mathematics, Peking University, Beijing, 100871 China

Current address: Fakultät und Institut fúr Mathematik, Ruhr-Universität Bochum, Postfach 102148, 4630 Bocum 1, Germany

Department of Mathematics, University of California, Los ANGeles, California 90024 Current address: Department of Mathematics, East China Normal University, Shanghai, 200062 China 